\title{
As Relações entre Autismo, Comportamento Social e Função Executiva
}

\author{
Cleonice Alves Bosa ${ }^{2}$ \\ Universidade Federal do Rio Grande do Sul
}

\begin{abstract}
Resumo
O objetivo do presente artigo é discutir o papel do lobo frontal nos comportamentos que caracterizam a síndrome do autismo. Focaliza, em especial, as possíveis relações entre função executiva, teoria da mente e habilidade de atenção compartilhada. Conclui-se que, apesar da investigação sobre a hipótese de comprometimento da função executiva como déficit subjacente ao autismo ser uma área promissora, muitas questões ainda permanecem abertas, como por exemplo, a questão da relação causal função executiva-déficit social; dificuldades acerca da especificidade deste comprometimento na área do autismo; a necessidade de investigar-se a natureza e intensidade do comprometimento na função executiva dentro dos subgrupos que compõem o espectro autista e a sobreposição dessa teoria com a de coerência central. Argumenta-se que a discussão sobre relações entre função executiva e comportamento social será incompleta caso não for compreendida a partir de um contexto psicossocial no qual se inserem os indivíduos com autismo e suas famílias.

Palavras-chave: Autismo; função executiva; lobo frontal; comportamento social.
\end{abstract}

\section{The Relationship between Autism, Social Behaviour and Executive Function}

\begin{abstract}
The aim of this paper is to discuss the role of the frontal lobe on the behaviours which characterize the autistic syndrome. The possible relationship between the executive function, theory of mind, and joint attention ability is specially focused. It is concluded that although the investigation of the hypothesis of the executive function underlying the deficts in autism is a promissing area, there are many questions which remain open, such as the causal executive function-social deficit relationship; difficulties regarding the restriction of this deficit to the autism; the need of investigating both the nature and the intensity of the executive function deficits within the subgroups which compound the autistic spectrum area, and the overlap between this theory and the central coherence theory. It is argued that the discussion about the relationship between the executive function and social behaviour will be incomplete if it does not take place within the psychosocial context in which the individuals with autism and their families are inserted.

Keywords: Autism; executive function; frontal lobe; social behaviour.
\end{abstract}

A teoria do lobo frontal aplicada ao autismo sugere que muitas das características dessa síndrome, como por exemplo, inflexibilidade (expressa através de atividades ritualizadas e repetitivas), perseveração, foco no detalhe em detrimento de um todo, dificuldade em gerar novos tópicos durante o brinquedo de faz-de-conta e dificuldades no relacionamento interpessoal, podem ser explicadas por comprometimento no funcionamento do lobo cerebral frontal (Duncan, 1986). Tal área ocupa 1/3 do cérebro humano e é responsável pela execução de atividades a partir de informações recebidas pelas regiões

\footnotetext{
${ }^{1}$ Endereço para correspondência: Departamento de Psicologia do Desenvolvimento e da Personalidade, Instituto de Psicologia, UFRGS, Rua Ramiro Barcelos, 2.600, Porto Alegre, RS, 90035003. E-mail: cleobosa@conex.com.br

${ }^{2}$ Parte desse artigo deriva da tese de doutorado da autora, realizada no Institute of Psychiatry, Londres, UK, subsidiada pelo CNPq.
}

posteriores do córtex. As partes posteriores compreendem regiões responsáveis pela informação sensorial enquanto a parte anterior (pré-frontal) organiza as informações emotivas, mneumônicas e da atenção, oriundas do sistema límbico ou do cerebelo, além das sensoriais. Mais especificamente, essa região é responsável pela capacidade de planejamento e desenvolvimento de estratégias para atingir metas, o que requer flexibilidade de comportamento, integração de detalhes num todo coerente e o manejo de múltiplas fontes de informação, coordenados com o uso de conhecimento adquirido (Kelly, Borrill \& Maddell, 1996).

A hipótese de comprometimento da função executiva como déficit subjacente ao autismo surgiu em função da semelhança entre o comportamento de indivíduos com disfunção cortical pré-frontal e daqueles com autismo (Duncan, 1986). Pessoas que sofreram lesões nas regiões pré-frontais tenderam a apresentar alterações de 
personalidade, tais como aumento da irritabilidade ou, ao contrário, apatia; perda do juízo crítico envolvendo valores sociais, além de problemas na área da atenção, memória de trabalho e prospectiva (Eslinger \& Damasio, 1985). As falhas na memória prospectiva podem ser explicadas tanto por uma ruptura entre as informações emocionais oriundas do sistema límbico e as informações objetivas dos sistemas sensoriais quanto por problemas na planificação de ações complexas futuras. Cabe então salientar que, embora a definição de função executiva refirase, prioritariamente, à habilidade no planejamento de estratégias de resolução de problemas para a execução de metas, mediada pelo córtex frontal (Luria, 1981), há clara intersecção com a capacidade de atenção e memória (Bebko \& Ricciuti, 2000). Na verdade, tem sido controversa a questão da inclusão de habilidades de memória de trabalho e processos inibitórios no conceito de função executiva, conforme observaram alguns autores (Dennis, 1991; Goldman-Rakic, 1987). Essa questão foi mais detalhadamente examinada por Nydén, Gillberg, Hjelmquist e Heiman (1999), os quais apontaram o problema dos limites entre a psicologia cognitiva e a neuropsicologia no que se refere ao estudo da função executiva. Esses autores observaram que os termos metacognição e função executiva têm sido usados indiscriminadamente para descrever processos envolvendo seleção, controle e monitorização no uso de estratégias cognitivas e criticaram a amplitude do conceito. Ressaltaram a urgência da necessidade de decomposição das funções executivas complexas em aspectos mais específicos (Ex: modalidades sensoriais, tempo de reação, etc.), conforme os modelos de processamento da informação, preenchendo, então, a lacuna entre essas duas áreas do conhecimento. De forma similar, Ozonoff, Pennington e Rogers (1991) também apontaram que uma das limitações dos testes comumente utilizados para medir a função executiva (Ex: Wisconsin Card Sorting Test, Heaton 1981) é justamente a impossibilidade de decomposição de funções cognitivas complexas em unidades elementares. Isso permitiria a identificação de comprometimento em funções específicas e a investigação da associação entre essas funções e diferentes patologias, como por exemplo, esquizofrenia e déficit de atenção e hiperatividade.

As semelhanças entre pessoas com autismo e aquelas com lesão frontal foram subseqüentemente comprovadas pelos resultados do desempenho de indivíduos com autismo em testes destinados a medir funções executivas (Hughes \& Russel, 1993; Ozonoff e cols., 1991). O interessante desses estudos é que foram realizados com crianças, demonstrando evidências contrárias à idéia de que as áreas pré-frontais fossem não-funcionais até a adolescência. Essa noção já havia sido desafiada por autores chamando a atenção para a emergência de certos aspectos dessa função, ainda no primeiro ano de vida da criança (Diamond, 1988; Diamond \& Goldman-Rakic, 1989).

Hughes e Russel (1993) utilizaram um tipo de experimento no qual a criança deveria aprender a obter bolinhas de gude de dentro de uma caixa, utilizando-se de uma entre duas diferentes estratégias. As crianças com autismo, comparadas aos grupos de controle, falharam em aprender a forma correta para obter esse fim, demonstrando maior insistência na estratégia incorreta e evidenciando um déficit maior na capacidade de planejamento para atingir uma meta.

Ozonoff, Strayer, McMahon e Filloux (1994) compararam um grupo de crianças/adolescentes diagnosticadas como tendo autismo - pelos critérios do DSM IV (APA, 1994) - com outros dois grupos de controle (síndrome de La Tourette e desenvolvimento típico), utilizando-se do paradigma do processamento da informação. O grupo com autismo obteve um desempenho comparável aos grupos de controle em tarefas que exigiam processamento global/local (atenção ao detalhe ou ao todo) e inibição de respostas a estímulos neutros, o que não ocorreu nas tarefas que requeriam flexibilidade cognitiva (mudança de foco de atenção de um padrão de estímulo para outro), reforçando a noção de disfunção executiva na síndrome do autismo.

Por outro lado, Griffith, Pennington, Wehner e Rogers (1999) desafiaram essa noção, pelo menos no que diz respeito a crianças pré-escolares. Os dois estudos reportados por eles (um transversal e outro longitudinal), utilizando as mesmas crianças dessa faixa etária, falharam em distinguir o grupo com autismo de um grupo de controle em tarefas visando a medir a função executiva. De modo similar, Nydén e colaboradores (1999), ao compararem grupos de crianças com síndrome de Asperger, com transtornos na leitura e escrita e um grupo não-clínico, em tarefas que avaliavam a função executiva, não conseguiram identificar um padrão específico a qualquer um dos grupos clínicos; todos eles apenas diferiram significativamente do não-clínico. O intrigante neste estudo é que o grupo que apresentou comprometimento mais severo e consistente entre as diferentes medidas utilizadas foi o de déficit de atenção e hiperatividade e não o de síndrome de Asperger como se esperaria, já que este último pertence ao espectro autista, de acordo com o DSM-IV. Como exemplo dessas medidas cita-se o Wisconsin Card Sorting Test, Vai-nãovai e teste do Conflito (Becker, Isaac \& Hynd, 1987; 
Mühlenbock \& Heiman, 1995). Embora reconhecendo a necessidade de replicação desses estudos para que a questão do comprometimento da função executiva no autismo seja esclarecida, é importante ressaltar que tais discussões geraram um outro campo de investigação, qual seja, o da relação entre função executiva e comportamento social.

\section{A Relação Entre Função Executiva e Comportamento Social}

Uma das primeiras áreas do desenvolvimento a eliciar preocupação nos cuidadores das crianças diagnosticadas como tendo autismo é a de comunicação e interação social, ainda nos dois primeiros anos de vida da criança (Lord, Storoschuk, Rutter \& Pickles, 1993). Diversos estudos têm documentado o comprometimento de crianças com autismo quanto à atenção compartilhada, definida como a habilidade envolvendo a alternância do olhar e outros sinais comunicativos entre o parceiro e o objeto/evento, que é o foco de atenção da criança (ver Mundy \& Sigman, 1989 para uma revisão). A importância de estudos na área de atenção compartilhada e dos mecanismos envolvidos nessa habilidade, como por exemplo, a função executiva, atrela-se a sua possível condição de precursora da capacidade de desenvolver uma "teoria da mente", cuja discussão será retomada mais adiante.

\section{Atenção Compartilhada}

Um dos primeiros estudos a focalizar a relação entre lobo frontal e atenção compartilhada foi o realizado por McEvoy, Rogers e Pennington (1993). Esses autores demonstraram que o grupo de crianças pré-escolares com autismo comparados aos grupos de controle apresentou a mesma tendência de perseveração na estratégia incorreta em uma tarefa de reversão espacial, utilizada para medir função executiva. A performance nessa tarefa correlacionou-se positivamente com a habilidade no comportamento de atenção compartilhada, sugerindo que essa habilidade pode estar relacionada à maturação dos lobos frontais. Ambas as habilidades emergem no mesmo período, isto é, no segundo semestre de vida do bebê. $\mathrm{Na}$ verdade, Mirsky (1987), utilizando-se do modelo de processamento da informação, sugeriu que o processo de atenção envolve quatro componentes independentes (focalização, sustentação, deslocamento e decodificação da atenção) os quais localiza-se em diferentes áreas cerebrais. Frisou que, desses componentes, somente a capacidade de mudar o foco de atenção de maneira adaptativa (shift attention) seria função do córtex préfrontal. Essa habilidade foi investigada, mais Psicologia: Reflexão e Crítica, 2001, 14(2), pp. 281-287 especificamente, no estudo de Belmonte (2000). Esse autor empregou medidas eletrofisiológicas de velocidade e especificidade de atenção em oito adultos com diagnóstico de autismo e um grupo de controle. Os resultados apontaram para uma anormalidade no processo de divisão da atenção frente a estímulos, caracterizada por uma falha dos dois hemisférios cerebrais em operar de forma independente. Esses resultados foram consistentes com relatos prévios de perda de especialização hemisférica no autismo (Dawson \& Lewy, 1989). Essa abordagem explica os sintomas autísticos (Ex: retraimento social e estereotipias) como resultado de uma dificuldade em modular a experiência sensorial. Dessa forma, pessoas com autismo experienciariam uma sobrecarga sensorial durante a interação social, considerando-se que o ser humano é uma das fontes mais ricas de estimulação simultânea: tom da vOz (estímulo auditivo), expressão facial (estímulo visual), gestos (estímulo visual periférico) e referência a objetos e eventos ao redor (estímulo visual e auditivo periférico). O retraimento social e as estereotipias seriam formas de fugir dessa sobrecarga. $\mathrm{O}$ autor refere que, de acordo com princípios da fisiologia, o comportamento obsessivo é explicado em termos de um retorno a comportamentos mais simples, os quais são repetidos incansavelmente, como forma de lidar com a disfunção atencional.

A dificuldade de pessoas com autismo para lidar com as demandas atencionais de estímulos visuais e auditivos, simultaneamente, também foi o foco dos estudos de Courchesne e colaboradores (1994). Os autores demonstraram que adolescentes com autismo apresentaram um comprometimento na capacidade de responder a diferentes modalidades de estímulos, quando estas eram apresentadas em um intervalo de tempo inferior a 2,5 segundos. O mesmo problema evidenciavase diante da necessidade de dividir atenção entre diferentes propriedades de um mesmo estímulo (ex: cor e forma). Tal desempenho não poderia ser atribuído à deficiência mental, uma vez que essa variável foi controlada (todos os participantes apresentavam QI superior a 70).

McEvoy e colaboradores (1993) levantaram as seguintes hipóteses para a relação entre atenção compartilhada e função executiva: a) a habilidade cognitiva de mudanças no foco de atenção é essencial para o desenvolvimento da capacidade de atenção compartilhada; b) os processos de atenção compartilhada são importantes para o desenvolvimento da habilidade cognitiva de mudanças no foco de atenção; c) a relação entre atenção compartilhada e habilidade cognitiva de mudanças no foco de atenção é mediada por um terceiro fator (lobo frontal) o qual é comum a ambas as 
habilidades, e d) há uma causação recíproca e complexa entre esses dois processos. De qualquer modo, a premissa da habilidade de atenção compartilhada, enquanto precursora de outra - a da teoria da mente - incrementou os estudos nessa área.

\section{Função Executiva (FE) e Teoria da Mente (ToM)}

Teoria da Mente tem sido definida como a capacidade para atribuir estados mentais (crenças, desejos, conhecimento e pensamentos) a outras pessoas e predizer o comportamento das mesmas em função destas atribuições (Baron-Cohen, Leslie \& Frith, 1985). A compreensão da criança a respeito das crenças dos outros foi primeiro investigada, experimentalmente, por Wimmer e Perner (1983), utilizando-se de um teste baseado numa estória de bonecos, na qual um personagem mantém uma crença falsa (diferente) daquela da criança. Crianças que passavam neste teste demonstravam capacidade para predizer o comportamento do personagem baseada na crença (falsa) do mesmo. Baron-Cohen e colaboradores (1985) adaptaram este experimento, criando o teste da Sally-Ann, para investigar o possível comprometimento de crianças com autismo na habilidade de usar o contexto social para compreender o que outras pessoas pensam e acreditam. Essas crianças demonstraram dificuldades em compreender o que o personagem pensava e em predizer o seu comportamento com base no seu pensamento. Tais resultados foram replicados, subseqüentemente (Ozonoff, Pennington \& Rogers, 1991; Prior, Dahlstrom \& Squires, 1990), levando à conclusão de que crianças com autismo apresentam um atraso ou desvio no desenvolvimento da capacidade de meta-representar, isto é, desenvolver uma 'teoria da mente' (Baron-Cohen, 1993). Este comprometimento acarretaria déficits no comportamento social como um todo e na linguagem. Os déficits de linguagem seriam uma conseqüência da incapacidade destas crianças para se comunicarem com outras pessoas a respeito de estados mentais; os distúrbios no comportamento social refletiriam a dificuldade em dar um sentido ao que as pessoas pensam e ao modo como se comportam. Denominou esse comprometimento de "cegueira mental". Esta teoria afirma que aqueles comportamentos sociais que não envolvem metarepresentação (Ex: comportamentos afiliativos e de apego) podem estar relativamente intactos.

Bailey, Philips e Rutter (1996) discutiram a controvérsia acerca da relação entre comportamento social e função executiva, a qual girou em torno de uma direção causal se é o comprometimento na habilidade de metarepresentar que afeta a função executiva ou o contrário. A favor da primeira direção causal está o argumento de que a compreensão das intenções do outro é fundamental para a função executiva. Entretanto, os autores referem que essa possível relação tem sido raramente investigada e citaram apenas um estudo que se ocupou dessa questão (Ozonoff, Pennington \& Rogers, 1991). Contudo, o resultado foi contrário à causação ToM-FE uma vez que pessoas com autismo falharam nas tarefas de função executiva mesmo tendo passado naquelas utilizadas na mensuração da teoria da mente (tarefas da falsa crença). Entretanto, chamaram a atenção para os problemas metodológicos que possam ter influenciado os resultados como a falta de variabilidade nas respostas à tarefa de ToM (efeito de teto) e a confusão conceitual entre habilidade de compreensão de falsas crenças nos outros e suas próprias intenções. A favor da segunda posição (causação FE -ToM) está o argumento de que a resolução das tarefas de ToM requer habilidades cognitivas gerais como, por exemplo, memória de trabalho, inibição de respostas irrelevantes e inferência. Portanto, a falha nas tarefas de ToM seria decorrente de uma incapacidade para lidar com as demandas da tarefa que requerem função executiva (planejamento e flexibilidade). Os autores contra-argumentaram, apresentando evidências de um estudo que empregou tarefas de controle (Sodian \& Frith, 1992, citado em Bailey e cols., 1996) no qual medidas de função executiva não correlacionaram-se com as de ToM. Mais especificamente, sucesso nas tarefas de FE não levou igualmente ao sucesso nas tarefas de ToM. Os autores concluíram que o autismo pode ser resultante de comprometimentos em sistemas neurais diferentes e relativamente independentes: um responsável pelo funcionamento global da função executiva, e outro modular, especificamente orientado para a interação social.

Baron-Cohen (1995) faz uma ressalva em relação aos seus próprios postulados como explicação única para a síndrome do autismo e sugere modelos explicativos como complementos aos seus: "Eu mencionei anteriormente os indivíduos os quais sofrem tanto de cegueira da mente quanto de comprometimento no sistema executivo. De fato, essa combinação parece ser substancial no autismo, o primeiro produzindo comportamentos repetitivos, rígidos e inflexíveis, associados ao autismo, e o segundo produzindo as anormalidades sociais e comunicativas as quais constituem seus marcadores.” (p. 137)

Finalmente, outra área de estudos que está começando a receber maior atenção empírica é a que busca investigar a associação entre brinquedo simbólico e função executiva Jarrold, Boucher \& Smith, 1996; Jarrold, Carruthers, Smith \& Boucher, 1994). Tal tarefa é interessante pela relação entre brinquedo simbólico e teoria da mente. Um aspecto que vale a pena ressaltar é o desafio que Jarrold 
e colegas fazem à tese de Leslie (1987) de que o comprometimento na capacidade de metarepresentação é subjacente ao déficit no brinquedo de faz-de-conta e na habilidade para desenvolver uma teoria da mente. De acordo com Leslie, o brinquedo simbólico é a manifestação mais precoce da capacidade de metarepresentar - definida como a habilidade de atribuir estados mentais ao self e a outros (Premack \& Woodruff, 1978). Para Jarrold e colaboradores (1996), o comprometimento quanto a esse tipo de brinquedo residiria mais na dificuldade em gerar novos atos (capacidade ligada à função executiva) do que em produzir atos isolados de brinquedo funcional (ex: colocar a xícara no pires) ou de substituição (ex: usar um bloco de madeira como telefone), evidência de capacidade metarepresentacional. De fato, outros estudos também demonstraram a presença de brinquedo funcional e de substituição em crianças com autismo (Charman \& Baron-Cohen, 1997; Lewis \& Boucher, 1988), principalmente quando auxiliadas por um adulto em situações estruturadas. Contudo, a freqüência destes atos foi menor do que a de grupos de controle (crianças com desenvolvimento típico ou com deficiência mental, emparelhadas por idade mental). De qualquer modo, é evidente a gama de modelos explicativos para os comprometimentos e habilidades observados em pessoas com autismo, ocorrendo, inclusive, sobreposição entre as mesmas.

\section{A Sobreposição entre Função Executiva e Coerência Central}

Tanto Bailey e colaboradores (1996), quanto BaronCohen (1995), mencionam uma outra explicação para os comprometimentos observados na síndrome do autismo: o drive para coerência central (Frith, 1989), o qual encontra-se comprometido no autismo e que parece ser independente da cegueira mental e da função executiva. A falta da tendência natural em juntar partes de informações para formar um 'todo' provido de significado (coesão central) é uma das características mais marcantes no autismo.

A tendência em ver partes, ao invés de uma figura inteira, e em preferir uma seqüência randômica, ao invés de uma provida de significado (contexto), pode explicar a performance superior de crianças com autismo nas escalas de Weschler que envolvem reunião e classificação de imagens por séries (Happé, 1994), nas tarefas de localização de figuras escondidas (Shah \& Frith, 1993) e de memorização de uma série de palavras sem-sentido ao invés daquelas com significado, comparadas aos controles (Hermelin \& O'Connor, 1970). Portanto, um dos aspectos interessantes dessa teoria é que focaliza tanto

Psicologia: Reflexão e Crítica, 2001, 14(2), pp. 281-287 as deficiências quanto as habilidades dos indivíduos com autismo. Em contrapartida, a sua relação com os déficits sociais e comunicativos não é esclarecida e é explicada apenas indiretamente, recorrendo à teoria da mente. Além disso, o destaque para o foco no detalhe, em detrimento do todo, também tem sido atribuído à função executiva, tornando obscura a suposta independência entre esses dois modelos.

\section{Considerações Finais}

Conclui-se que a investigação sobre a hipótese de comprometimento da função executiva como déficit subjacente ao autismo é uma área promissora. Entretanto, muitas questões ainda permanecem sem resposta. Os estudos acima revisados revelaram inconsistências quanto à especificidade deste comprometimento na área de autismo. Além disso, a natureza e intensidade do comprometimento na função executiva, dentro dos subgrupos que compõem o espectro autista (Ex: síndrome do X-frágil), também necessitam ser investigados para que a questão da especificidade seja esclarecida. A sobreposição dessa teoria com a da coerência central é outro aspecto que merece mais atenção, pois a questão da ortogonalidade entre os dois constructos tem implicações teóricas (os limites conceituais de cada um) e, conseqüentemente, empíricos (o risco de uma determinada tarefa medir ambas habilidades, simultaneamente). Entretanto, ambos os modelos dividem a mesma limitação para explicar a sua relação com o comportamento social, qual seja, a de recorrer a outros modelos (Ex: teoria da mente) ao tratarem da questão da intencionalidade no processo de interação social.

Chama-se, ainda, a atenção para peças desse quebracabeça que permanecem fora de encaixe. Por exemplo, como se justificaria o fato de uma criança com autismo apresentar tendência para coordenar o olhar e outros canais comunicativos entre o parceiro e objetos num contexto de busca de auxilio (ex: pedir para abrir a tampa de uma caixa de brinquedos) - indicativa de capacidade de modulação da atenção - porém, menos freqüentemente, num de compartilhamento de experiências sociais, conforme demonstrado por Bosa (1998). Essa autora recorreu à teoria afetiva contemporânea de Hobson (1993), o qual retoma a noção de Kanner de distúrbio inato do contato afetivo (Kanner, 1943) e à teoria da mente (Baron-Cohen, 1995) para explicar essa discrepância nos resultados. Hobson trata essa questão, sugerindo que pessoas com autismo desenvolvem, desde cedo, o conceito do outro como agente de ação, mas apresentam dificuldades em concebê- 
lo como agente de contemplação. A primeira habilidade relaciona-se muito provavelmente a questões de sobrevivência do indivíduo e a processos de aprendizagem. A segunda diz respeito ao desenvolvimento do conceito do outro como alguém que possui interesses similares acerca dos eventos e objetos no mundo, os quais são focalizados e compartilhados pelo simples prazer do compartilhamento. A teoria afetiva e a teoria da mente sobrepõem-se quanto a esse aspecto.

A literatura na área da psicologia do desenvolvimento é farta em evidências sobre a capacidade do bebê, ainda no primeiro ano de vida, para dirigir a atenção do outro em situações de exploração e descobertas do ambiente (ver Messer, 1994, para uma revisão mais completa). $\mathrm{Na}$ verdade, os esforços do bebê para buscar a proximidade de contato físico com o cuidador e manter a interação social com o mesmo, assim como as respostas do cuidador aos sinais infantis, são os fundamentos da teoria do apego de Bowlby (1969). Os postulados etológicos têm revertido em pesquisas desde a década de 60 até a atualidade, inclusive na área do autismo (ex: Capps, Sigman \& Mundy, 1994). De fato, essa área reveste-se de tal importância que a investigação sobre os comportamentos de apego faz parte de um dos instrumentos mais utilizados na literatura para investigar os comprometimentos na área do autismo - o Autism Diagnostic Interview-Revised (Lord, Rutter \& LeCouteur, 1994).

Da mesma forma, a qualidade de interação social que se estabelece entre a criança e seus cuidadores também são informativas sobre o desenvolvimento social infantil, sendo sua importância amplamente documentada na área da psicologia do desenvolvimento (ver Bremmer, Slater \& Butterworth, 1997, para uma revisão da relação entre interação social e desenvolvimento infantil, incluindo processos de atenção). Existem evidências sobre a tendência de mães de crianças com autismo apresentar um estilo interativo extremamente diretivo ou, ao contrário, pouco estimulador (Trevarthen, 1996), dado o perfil social bizarro dessas crianças, assim como sobre a presença de estresse materno, em comparação a grupos de controle - ambos, fatores que podem influenciar a qualidade de interação cuidador-criança (Dumas, Wolf, Fisman \& Culligan, 1991; Fisman, Wolf \& Noh, 1989). Esses resultados apontam para a necessidade de levar-se em conta a influência de fatores psicossociais no desenvolvimento do comportamento social infantil. Em que medida esses processos interativos precoces influenciam o desenvolvimento dos processos de atenção e memória ou são influenciados por eles é que merecem ser mais amplamente investigados.
Enfim, concordando com Baron-Cohen (1995), parece-nos muito mais direta a relação entre déficit na função executiva (FE) e rigidez do comportamento (estereotipias e rotinas elaboradas, interesses circunscritos, etc.) do que entre FE e comportamento social. Longe de afirmar que essa relação não existe, ressalta-se a necessidade de mais investigações e a importância de compreender essa possível relação num contexto interativo, levando-se em conta aspectos psicossociais como possíveis mediadores das funções cerebrais.

\section{Referências}

American Psychiatric Association (1994). Diagnostic and statistical manual of mental disorders (4 ed.rev.; DSM-IV). Washington, DC: Author.

Bailey, A., Philips, W. \& Rutter, M (1996). Autism: Towards an integration of clinical, genetic, neuropsychological, and neurobiological perspectives. Journal of Child Psychology and Psychiatry, 37(1), 89-126.

Baron-Cohen, S. (1993). From attention-goal psychology to belief-desire psychology: The development of a theory of mind and its dysfunction. Em S. Baron-Cohen, H. Tager- Flusberg \& D. J. Cohen (Orgs.), Understanding other minds: Perspectives from autism. (pp. 59-82) Oxford: Oxford University Press.

Baron-Cohen, S. (1995). Mindblindness. Cambridge: MIT.

Baron-Cohen, S., Leslie, A. M. \& Frith, U. (1985). Does the autistic child have a 'Theory of mind'? Cognition, 21, 37-46.

Bebko, J. M. \& Ricciuti, C. (2000). Executive functioning and memory strategy use in children with autism: The influence of task constraints on spontaneous rehearsal. Autism, 4, 299-320.

Becker, M. G., Isaac, W. \& Hynd, G. W. (1987). Neuropsychological development of nonverbal behaviors attributed to 'frontal lobe functioning'. Developmental Neuropsychology, 3, 275-98.

Belmonte, M. (2000). Abnormal attention in autism shown by steady-state visual evoked potentials. Autism, 4, 269-285.

Bosa, C. (1998). Affect, social communication and self-stimulation in children with and without autism: a systematic observation study of requesting behaviours and joint attention. Dissertação de Doutorado não-publicada. Institute of Psychiatry, Universidade de Londres. Londres, Inglaterra.

Bowlby, J. (1969). Attachment and loss. Vol 1: Attachment. London: Hogarth. Bremmer, G., Slater, A. \& Butterworth, G. (1997). Infant development: Recent advances. East Sussex: Psychology Press.

Capps, L., Sigman, M. \& Mundy, P. (1994). Attachment security in children with autism. Development and Psychopathology, 6, 249-261.

Charman, T. \& Baron-Cohen, S. (1997). Prompted pretend play in autism. Journal of Autism and Developmental Disorders, 27, 325-332.

Courchesne, E., Townsend, J., Aksoomoff, N., Saitoh, O., Yeung- Courchesne, R., Lincoln, A.J., Haas, R., Schereibman, L. \& Lav, L. (1994). Impairment in shifting attention in autistic and cerebellar patients. Behavioral Neuroscience, 108, 848-65.

Dawson, G. \& Lewy, A. (1989). Arousal, attention, and socioemotional impairments of individuals with autism. Em G. Dawson (Org.), Autism: New perspectives on nature, diagnosis, and treatment (pp. 3-21). New York: Guilford.

Dennis, M. (1991). Frontal lobe function in childhood and adolescence: A heuristic for assessing attention regulation, executive control and the intentional states important for social discourse. Developmental Neuropschology, 7, 327-358.

Diamond, A. (1988). Differences between adult and infant cognition: Is the crucial variable presence or absence of language? Em L. Weiskrantz (Org.), Thought without language (pp. 337-370). New York: Oxford University. 
Diamond, A. \& Goldman-Rakic, P. S. (1989). Comparison of human infants and rhesus monkeys on Piaget's A not B task: Evidence for dependence on dorsolateral prefrontal cortex. Experimental Brain Research, 74, 24-40.

Dumas, J.E., Wolf, L.C., Fisman, S. \& Culligan, A. (1991). Parenting stress, child behavior problems, and dysphoria in parents of children with autism, Down Syndrome, behaviour disorders, and normal development. Exceptionality, 2(2), 97-110.

Duncan, J. (1986). Disorganization of behavior after frontal lobe damage. Cognitive Neuropsychology, 3, 271-290.

Eslinger, P. \& Damasio, A. R. (1985). Severe disturbance of higher cognition after bilateral frontal lobe ablation: Patient EVR. Neurology, 35, 17311741.

Fisman, S., Wolf, L. \& Noh, S. (1989). Marital intimacy in parents of exceptional children. Canadian Journal of Psychiatry, 34(6), 519-525.

Frith, U. (1989). Autism: Explaining the enigma. Oxford: Blackwell.

Goldman-Rakic, P. S. (1987). Circuitry of primate prefrontal cortex and regulation of behaviour by representational knowledge. Em F. Plum \& V. Mountcastle (Orgs.), Handbook of physiology (pp. 373-417). Bethesda: American Physiology Society.

Griffith, M., Pennington, B. F., Wehner, E. A. \& Rogers, S. J. (1999). Executive functions in young children with autism. Child Development, 70(4), 817832.

Happé, F. G. E. (1994). Autism: An introduction to psychological theory. London: UCL.

Heaton. R. K. (1981). Wisconsin Card Sorting Test Manual. Odessa, F.L. Psychological Assessment Resources.

Hermelin, B. \& O'Connor, N. (1970). Psychological experiments with autistic children. New York: Pergamon.

Hobson, P. (1993). Understanding persons: The role of affect. Em S. Baron-Cohen, H. Tager-Flusberg \& D. J. Cohen (Orgs.), Understanding other minds: Perspectives from autism (pp. 205-227). Oxford: Oxford Medical Publications.

Hughes, C. \& Russell, J. (1993). Autistic children's difficulty with disengagement from an object: Its implications for theories of autism. Developmental Psychology, 29, 498-510.

Jarrold, C., Carruthers, P., Smith, P. K. \& Boucher, J. (1994). Pretend play: Is it metarepresentational? Mind and Language, 9, 445-468.

Jarrold, C., Boucher, J. \& Smith, P. (1996). Generativity deficits in pretend play in autism. British Journal of Developmental Psychology, 14, 275-300.

Kanner, L. (1943). Affective disturbances of affective contact. Nervous Child, 2, 217-250

Kelly, T. P., Borrill, H. S. \& Maddell, D. L. (1996). Development and assessment of executive function in children. Child Psychology and Psychiatry Revien, 1, 46-51.

Leslie, A. M. (1987). Pretence and representations: The origins of 'theory of mind'. Psychological Review, 94, 412-426.

Lewis, V. \& Boucher, J. (1988). Spontaneous, instructed and elicited play in relatively able autistic children. British Journal of Developmental Psychology, 6, 325-339.
Lord, C., Storoschuk, S., Rutter, M. \& Pickles, A. (1993). Using the ADI-R to diagnose autism in preschool children. Journal of Infant Mental Health, 14(3), 234-252.

Lord, C., Rutter, M. \& Le Couter, A. (1994). Autism Diagnostic Interview - Revised: A revised version of a diagnostic interview for caregivers of individuals with possible pervasive developmental disorders. Journal of Autism and Development Disorders, 24, 659-686.

Luria, A. R. (1981). Fundamentos de neuropsicologia. São Paulo: Livros Técnicos e Científicos.

McEvoy, R. E., Rogers, S. J. \& Pennington, B. F. (1993). Executive function and social communication deficits in young autistic children. Journal of Child Psychology and Psychiatry, 34, 563-578.

Messer, D. (1994). The development of communication: From social interaction to language. Chichester: John Wiley \& Sons.

Mirsky, A. F. (1987). Behavioral and psychophysiological markers of disordered attention. Environmental Health Perspectives, 74, 191-199.

Mühlenbock, K. \& Heiman, M. (1995). Handledning till Backer's Visual and Auditory Testing. Topic data och Sprakbehandling HB.

Mundy, P. \& Sigman, M. (1989). Specifying the nature of the social impairment in autism. Em G. Dawson (Org.), Autism: New perspectives on nature, diagnosis, and treatment (pp. 3-21). New York: Guilford.

Nydén, A., Gillberg, C., Hjelmquist, E. \& Heiman, M. (1999). Executive function/attention deficits in boys with Asperger Syndrome, attention disorder and reading/writing disorder. Autism, 3, 213-228.

Ozonoff, S., Pennington, B. \& Rogers, S. (1991). Executive function deficits in high-functioning autistic individuals: Relations to the theory of mind. Journal of Child Psychology and Psycbiatry, 32, 1081-1105.

Ozonoff, S., Strayer, D. L., McMahon, W. \& Filloux, F. (1994). Executive function abilities in autism and Tourette Syndrome: An information processing approach. Journal of Child Psychology and Psychiatry, 35, 10151032.

Premack, D. \& Wooddruff, G. (1978). 'Does the chimpanzes have a theory of mind?' Behavioral and Brain Science, 1, 515-526.

Prior, M. R., Dahlstrom, B. \& Squires, T. L. (1990). Autistic children's knowledge of thinking and feeling states in other people. Journal of Child Psychology and Psychiatry, 31, 587-602.

Shah, A. \& Frith, U. (1993). An islet of ability in autistic children: A research note. Journal of Cbild Psychology and Psychiatry, 24, 613-620.

Trevarthen, C. (1996). Communicating and playing with an autistic child. Em C. Trevarthen, K. Aitken, D. Papoudi \& J. Robarts (Orgs.), Children with autism: Diagnosis and interventions to meet their needs (pp. 98-115). London: Jessica Kingsley.

Wimmer, H. \& Perner, J. (1983). Beliefs about beliefs: Representation and constraining function of wrong beliefs in young children's understanding of deception. Cognition, 13, 103-128.

Recebido: 28/08/2001

Revisado: 19/04/2001

Aceite final: $21 / 05 / 2001$

Sobre a autora:

Cleonice A. Bosa é Psicóloga, mestre em Psicologia do Desenvolvimento/UFRGS, Doutora em Psicologia pelo Instituto de Psiquiatria - Universidade de Londres/UK; Profa. Adjunto do PPG em Psicologia do Desenvolvimento/UFRGS. 